Journal of Engineering and Applied Sciences 14 (11): 3698-3706, 2019

ISSN: 1816-949X

(C) Medwell Journals, 2019

\title{
Modeling and Simulation of Tidal Energy
}

${ }^{1}$ Layth Mohammed Abd Ali, ${ }^{2}$ Haider Ahmed Mohmmed and ${ }^{3}$ Othman M. Hussein Anssari

${ }^{1}$ Quality Control Laboratory, University of Kufa, Najaf, Iraq

${ }^{2}$ College of Engineering, University of Kufa, Najaf, Iraq

${ }^{3}$ Information Technology Research and Development Center, University of Kufa, Najaf, Iraq

\begin{abstract}
The potential for generating electricity from tidal currents are massive it is a resource that is exploited for sustainable generation of electricity. High load operator creating from fluid characteristics and expectable resource properties making marine currents, especially, attractive for power obstetrics and useful matched to the other renewable energies. Furthermore, international conventions concerning climate controlled to the reemergence of renewable energy technology in the oceans. As a result, many demonstration schemas in tidal energy are designed to capture the tidal produced littoral currents. Study reviews the basic concepts of tidal power and reports on issues related to the topology of generators associated with tidal turbines. It is also making attempts to highlight future issues in order to catalog some of the emerging technologies fundamentally awarding to pertinent work that has been carry on wind turbines and on the ship fans. The study treats with the evolution model of MATLAB-Simulink to simulate current tidal turbines. Purpose of simulation model is to assess the potential for current tidal power generation.
\end{abstract}

$\underline{\text { Key words: Renewable energy, tidal energy, MATLAB Simulink, pertinent work, ship fans, power generation }}$

\section{INTRODUCTION}

The world is looking for alternative sources of fossil energy (the world depends on more than $90 \%$ of its electric energy on fossil sources), the aspirations for natural resources around us were the wind, the water, the Sun. The seas and oceans were the potential resource of renewable energy, the ocean movement carrying energy in a shape of the tides, waves and water flows. Some countries have used this new technology such as France, England and the United States. There is a constant interest in harnessing tidal energy and concentrating on estuaries where water crosses through narrow channels and increases flow speed. Scientists have resorted to the possibility of using coastal currents. This was a more effective option than its existence on estuaries (Chen et al., 2016).

The tidal energy is a type of motion energy which is saved in tidal currents that result from gravitation of the Sun and the Moon as well as the rotate of the Earth about its axis. Thus, this energy is classified as alternative energy.

Tidal energy is turn the tide into electrical energy. Most of the coastal states start to advantage from the tidal energy to produce electricity and then relieve pressure on thermic power stations and the outcome is to decrease pollution from oil-fired thermal plants (Efroimsky, 2018; Udo-Akuaibit, 2014). They can be classified into two types.

Hydropower: Dams that use the potential energy resulting from altitude difference between tides. This method suffers from problems of high cost of buildings in addition to the lack of suitable places widely and have negative effects on the environment as shown in Fig. 1, (Hashemi et al., 2015).

Tidal turbine energy: Taking advantage of the kinetic energy of sea waves, this method is used because of its low cost in addition to its least impact on the environment as shown in Fig. 2 (Abd Ali and Issa, 2018; Jahromi et al., 2013). Developments in turbine technology offer greater amounts of energy in the future, especially in the straits, estuaries or entrances to bays where water flow is high. The amount of water that moves the tide twice every day are huge and can be adapted to obtain a huge amount of energy up to $20 \%$ of the need of the United Kingdom. Tidal energy have some advantages such as:

A clean source and friendly to environment

Not affected by seasons and weather

Corresponding Author: Layth Mohammed Abd Ali, Quality Control Laboratory, University of Kufa, Najaf, Iraq 
- Because of its dependence on the Earth movement and a gravity of the Sun and the Moon it is a renewable energy

Production of electricity in high quantities (Astariz et al., 2015; Signorelli et al., 2012)

\section{MATERIALS AND METHODS}

Modelling of the system: The design of the turbine has a huge importance that limits the value of power from water stream (Fig. 3). This stream leads to turn on the turbine.

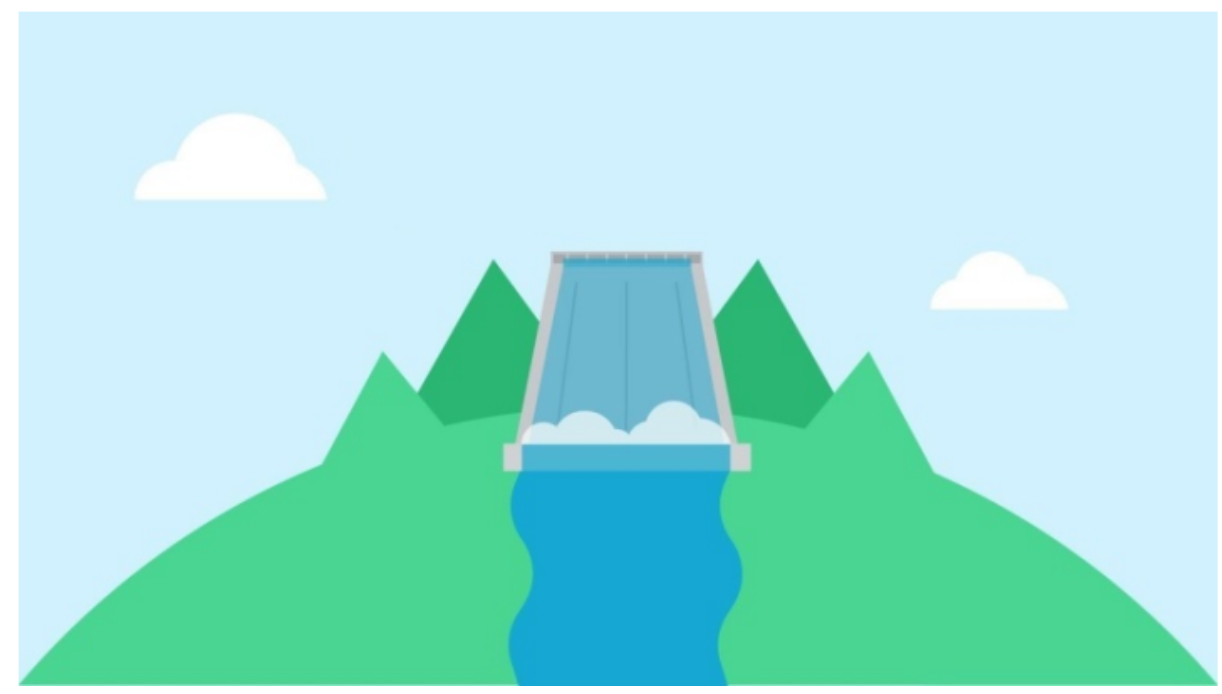

Fig. 1: Hydropower energy

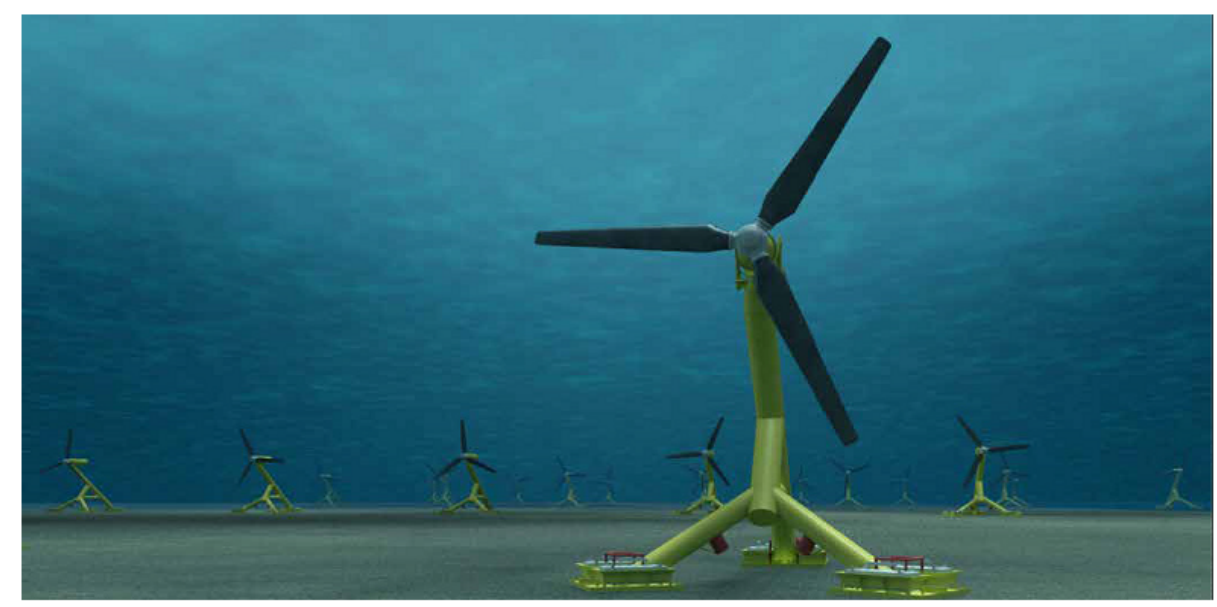

Fig. 2: Tidal turbines

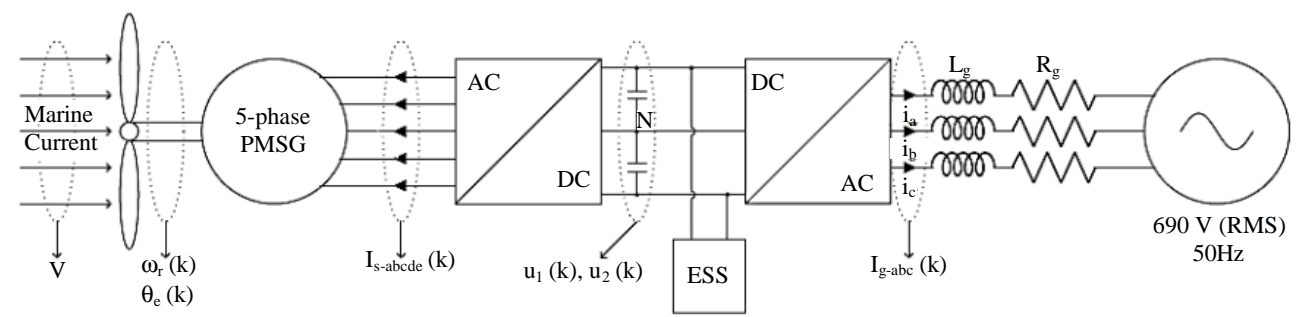

Fig. 3: The general plan of the water flow energy system 
The energy generated from the turbine depends on:

. The diameter of the armature

. The velocity of the tidal flow

Figure 3 represent the general plan of the water flow energy converter system and Fig. 4 and 5 represent the PMG controller and DC-controller,

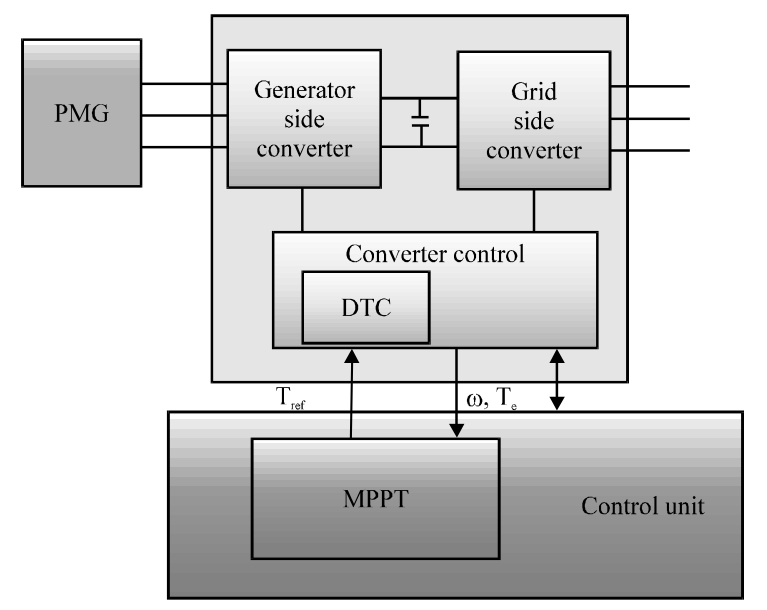

respectively (Ansari et al., 2018). The power factor $\mathrm{C}$, is variable and relies on the tip velocity ratio (2) and turbine slope bend (.) as shown in the Eq. 1 (Tallam et al., 2011):

$$
C_{p}(\lambda, \theta)=c_{1}\left(\frac{c_{2}}{\beta}-C_{3} \beta \theta-C_{4} \theta^{x}-C_{5}\right) e^{\frac{C_{6}}{\beta}}
$$

The characteristics $\curlywedge$, and, has been designed in MATLAB and execute as simulation showing in Fig. 6.

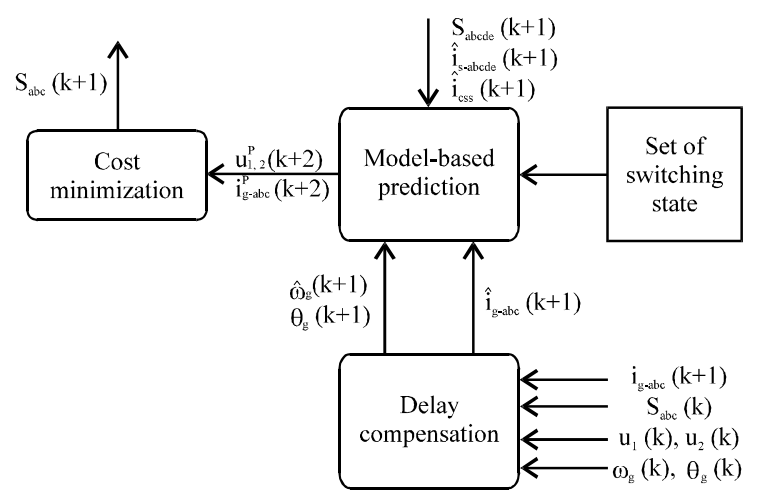

Fig. 4: The PMG controller

Fig. 5: The DC-controller

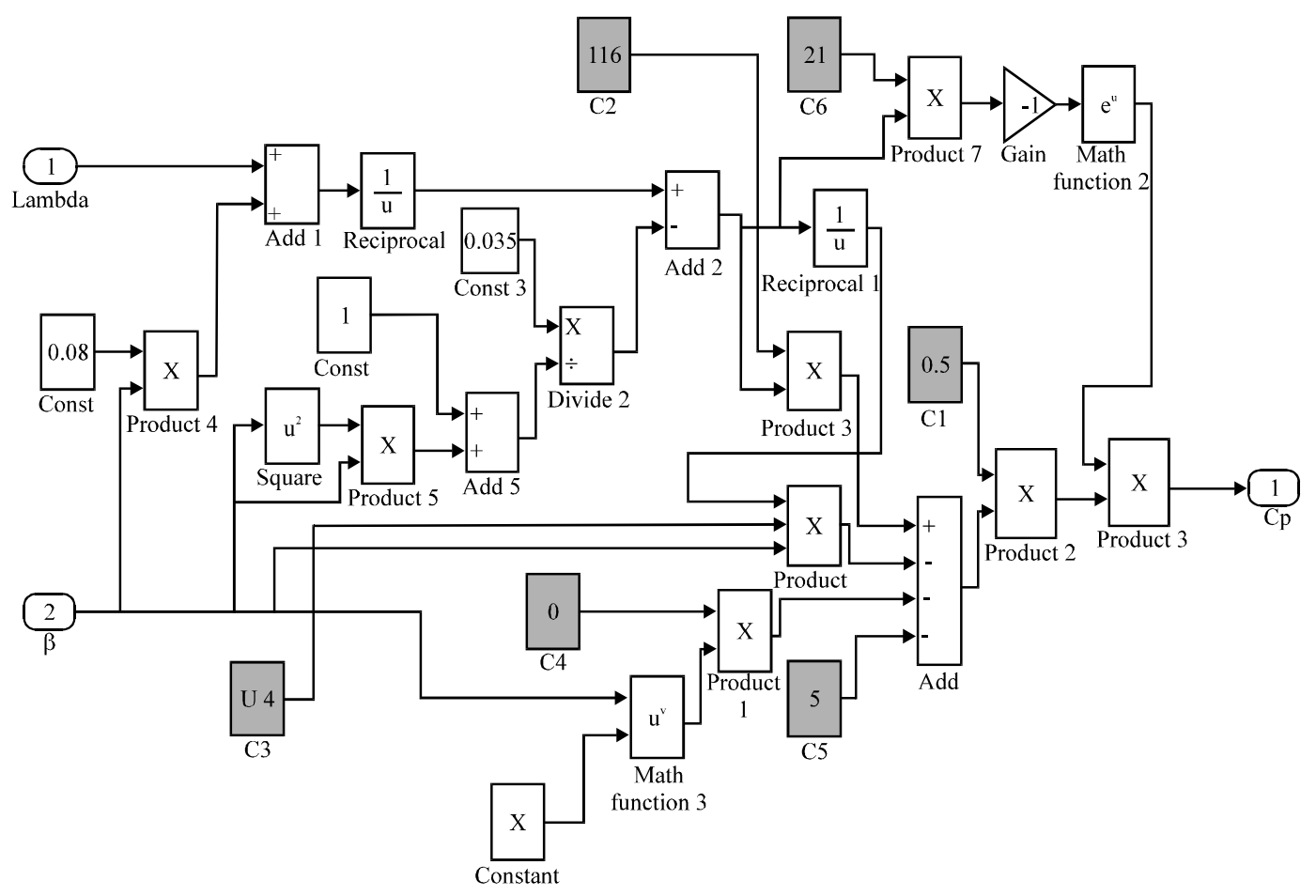

Fig. 6: The molding Simulation/MATLAB 


\section{RESULTS AND DISCUSSION}

In this part, various results in MATLAB/ Simulation are represented the power enhancement. These simulations have executed in MATLAB/Simulink medium. The IGBT switches are assumed typical. The 5-phase PMSG. The simulation period $(\mathrm{T})$ sample $=$ 9-5 sec. The simulation period $(\mathrm{T})$ sample $=9-5 \mathrm{sec}$. The system-jointed (5-phase) PMSG is established turbine tidal parameters have been recorded in an appendix Fig. 7 is shown the experimental tidal circle applied for results and Fig. 8 represent results of experimental for the of tidal, speed and tidal cycles at using an MPPT.

While the system work in a swell effect, $\mathrm{O} / \mathrm{P}$ power smoothing execution is evaluated. Figure 9-11 display active power inoculated into the grid while taking into consideration the swell effect. Simulations are

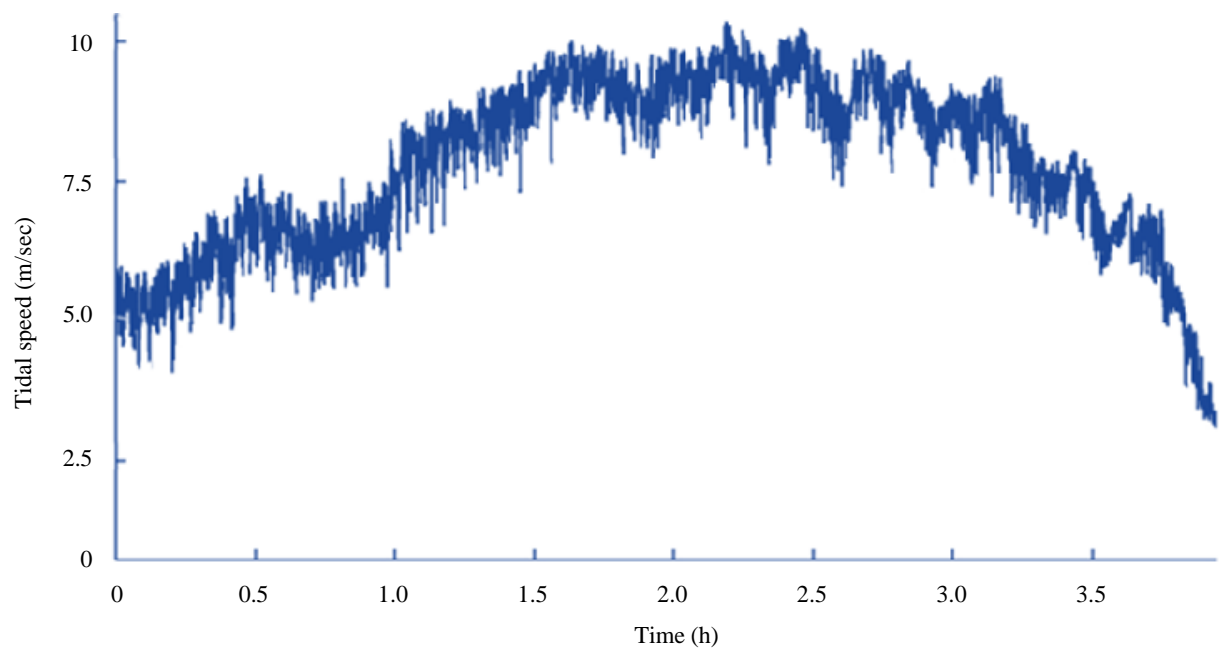

Fig. 7: Simulation of turbine by using tentative tidal currents data

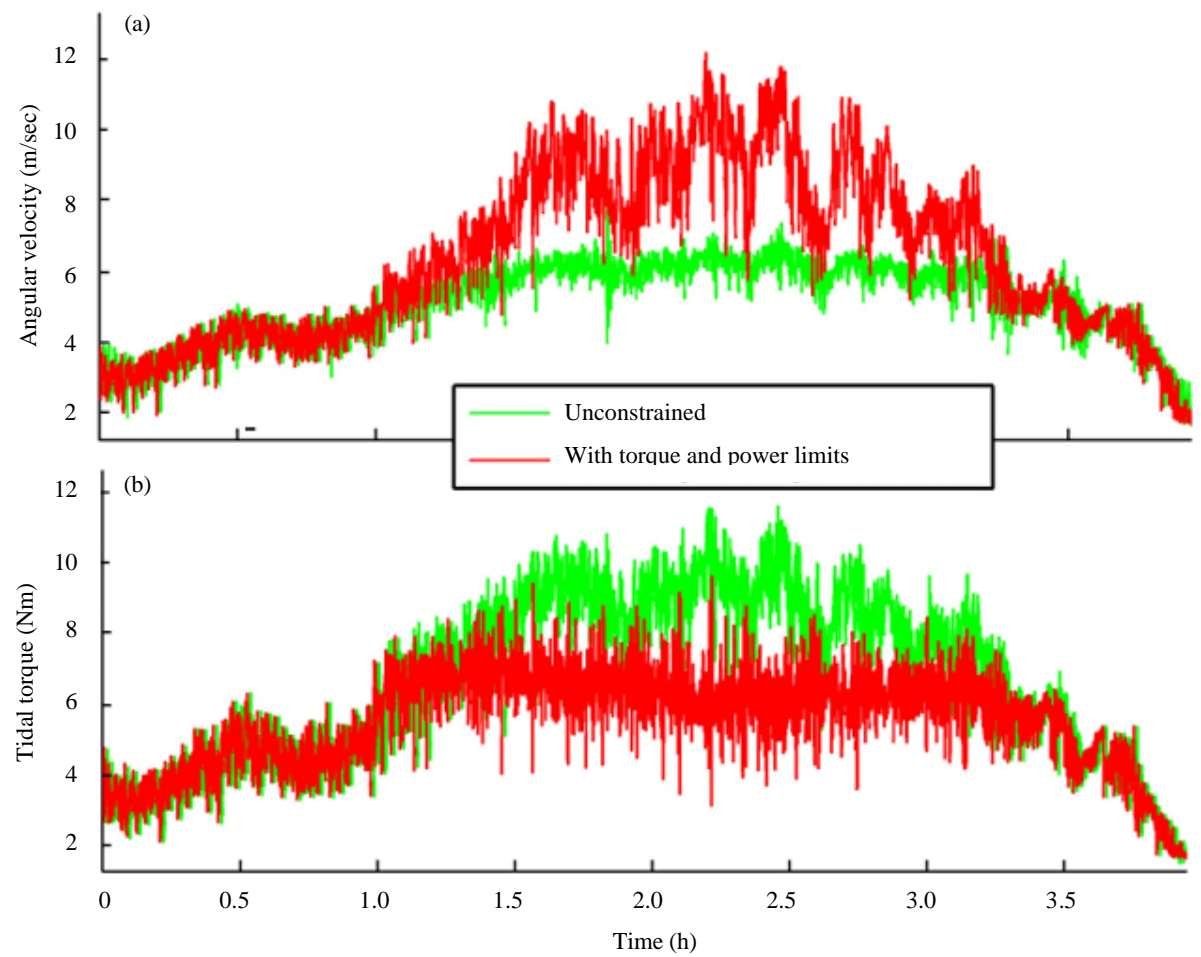

Fig. 8: a, b) The turbine operation at the lower velocity for the MPPT 

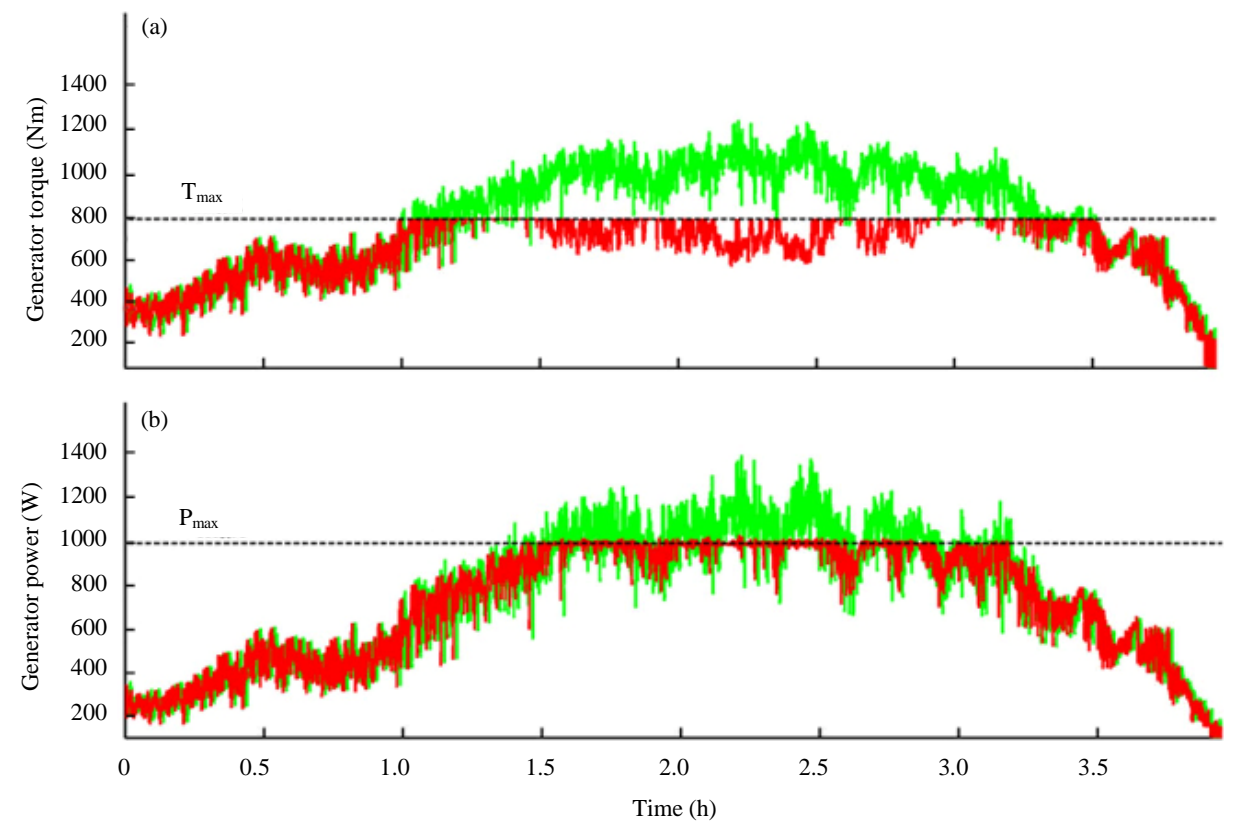

Fig. 9: a, b) The MPPT examined without and with torque chains and power

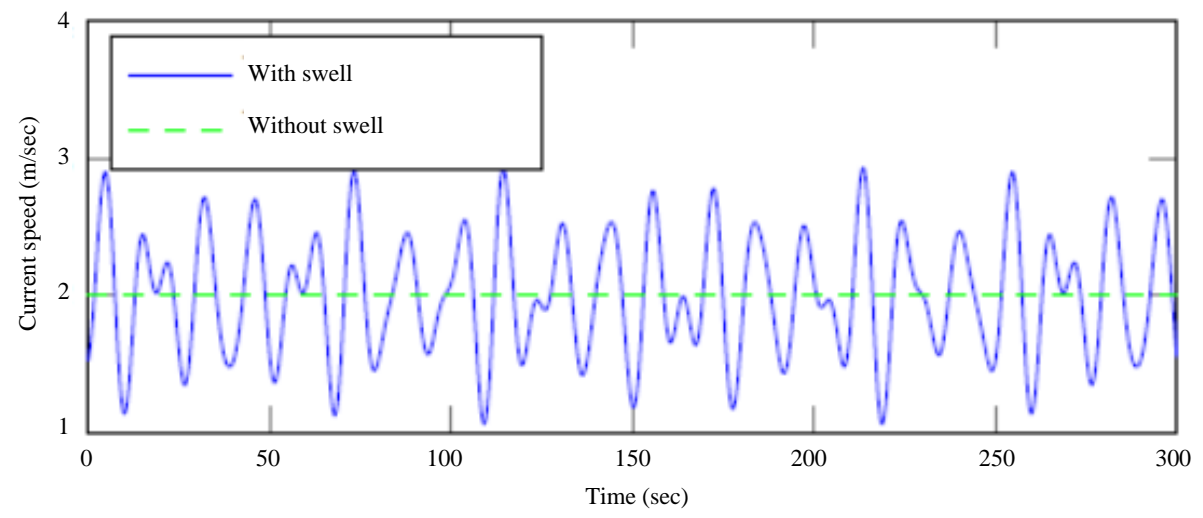

Fig. 10: Tidal velocity under the huge effect

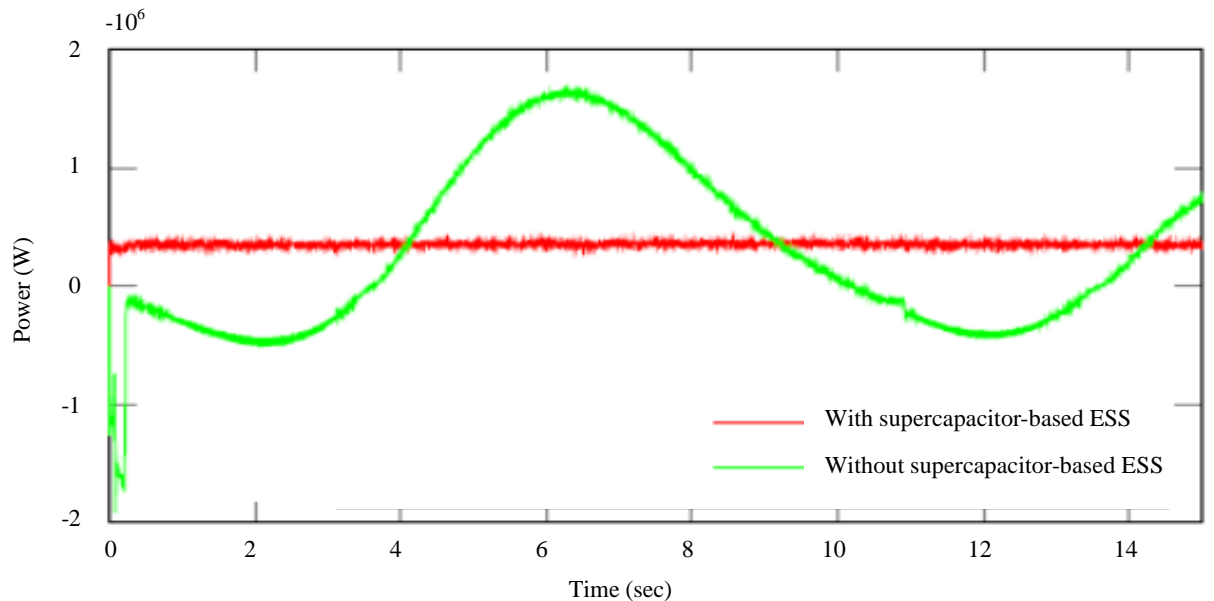

Fig. 11: Effective power without and with supper-capacitor established ESS 


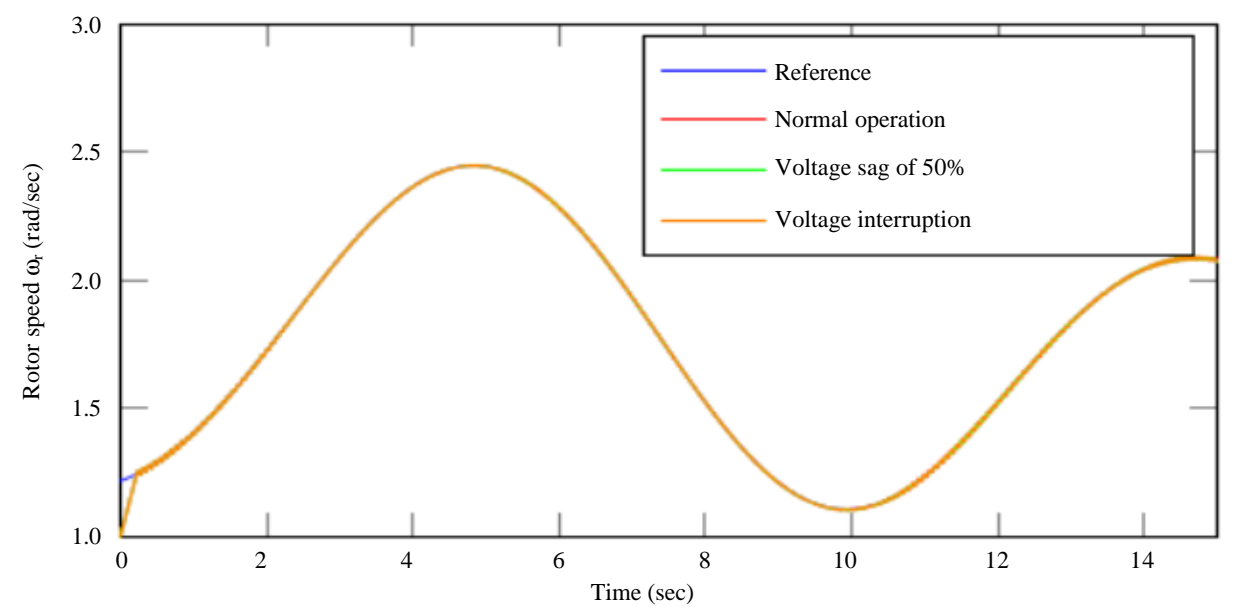

Fig. 12: Speed of rotor

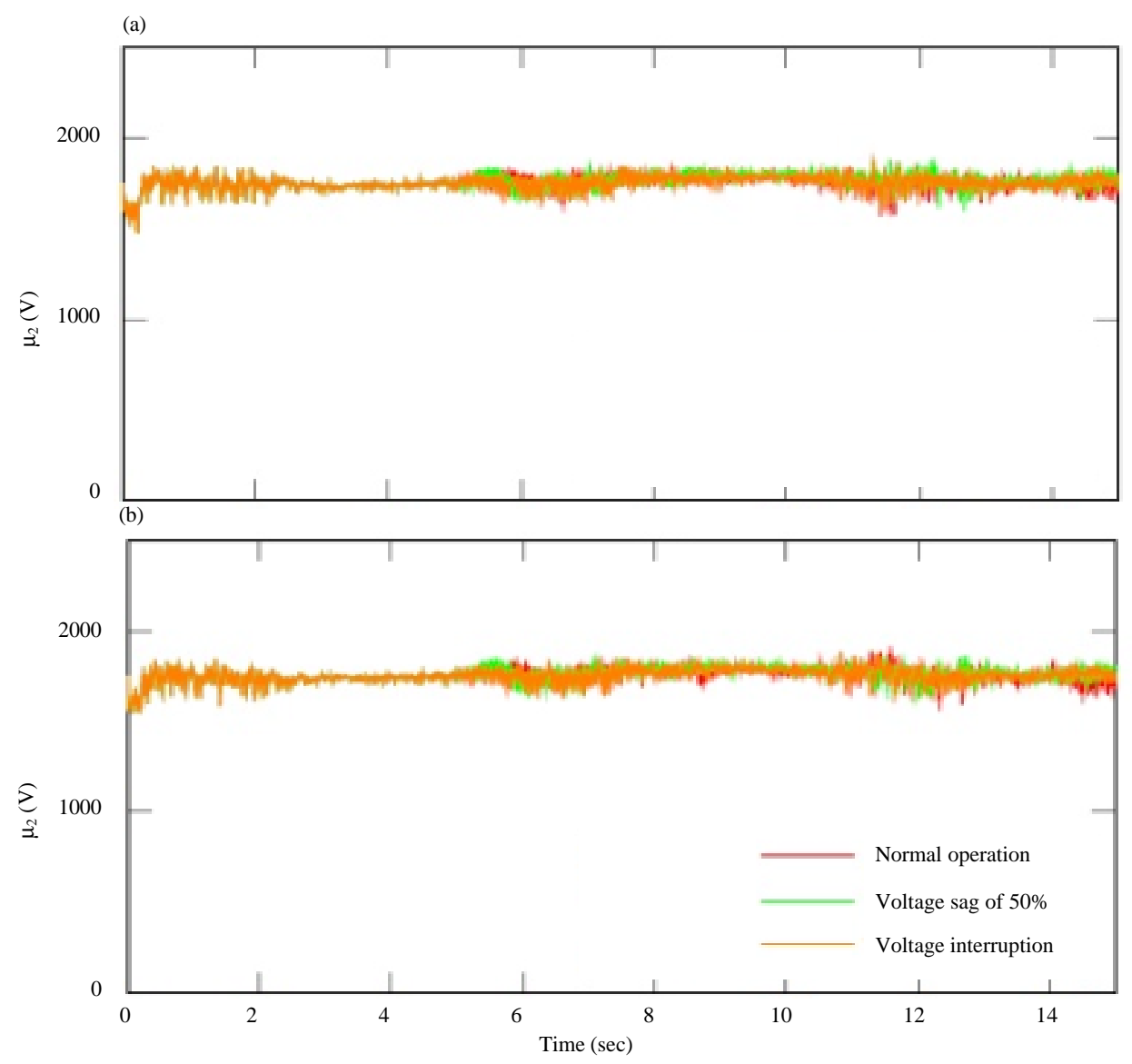

Fig. 13: a, b) Voltages for DC bus

also carried out in voltage sags. without cutting off the grid. Next 2 faults: grid voltages are decreased to $50 \%$ from the estimated value through $5 \mathrm{sec}(6-12 \mathrm{sec})$ voltages are quite interrupted through $0.3 \mathrm{sec}(5.5-5.8 \mathrm{sec})$ (Fig. 12-16).
Above-mentioned that faults period is 2 times lengthy the demands of grid blades. Figure 11 shows the 5-phase PMSG rotor speed. In both modes faulty and normal, the generator realizes good tracking executions. Tidal turbine residue at the maximum 
(a)
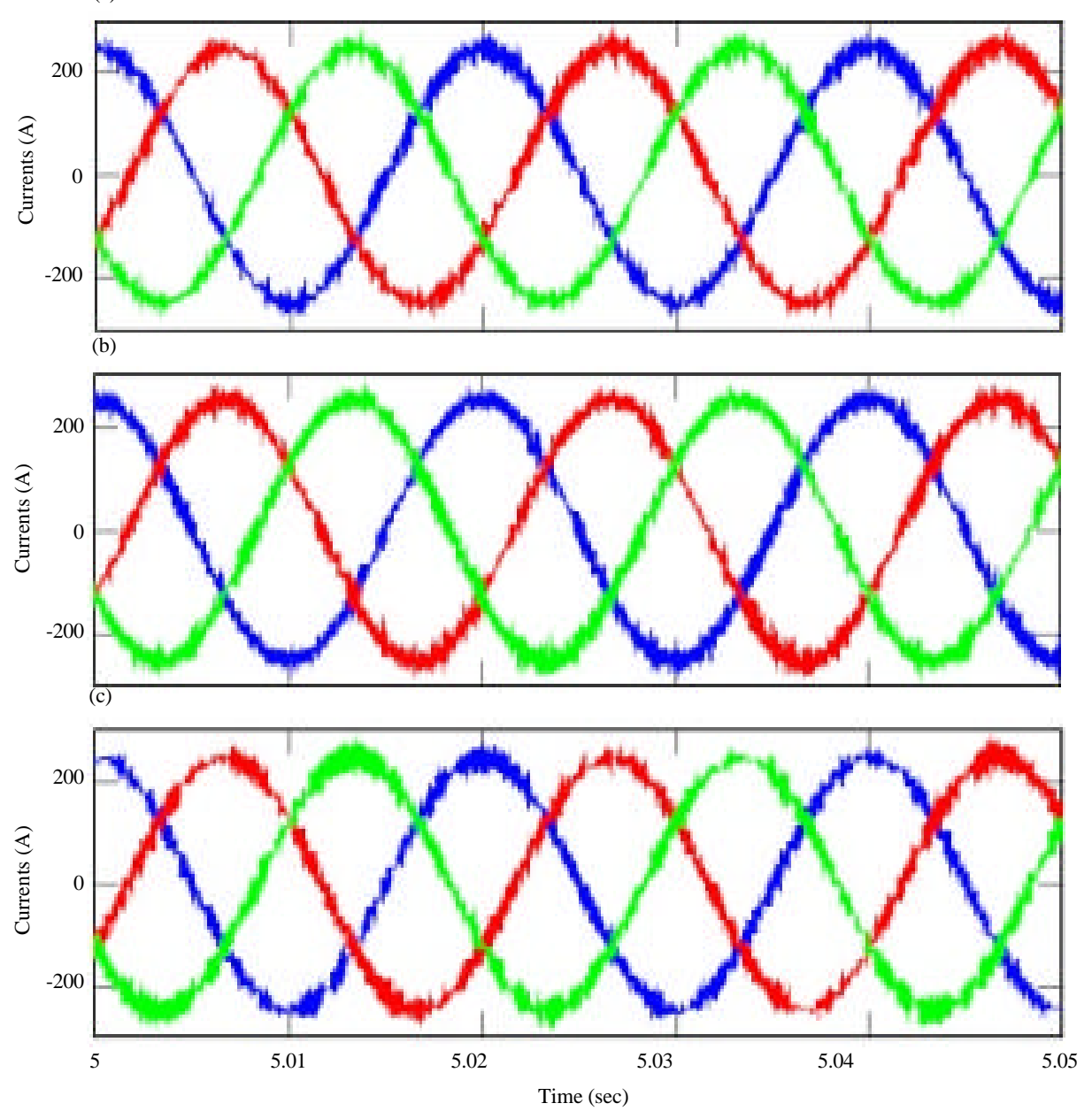

Fig. 14: a-c) Currents of grid for ordinary operation, voltage middle of $50 \%$ and voltage bottom

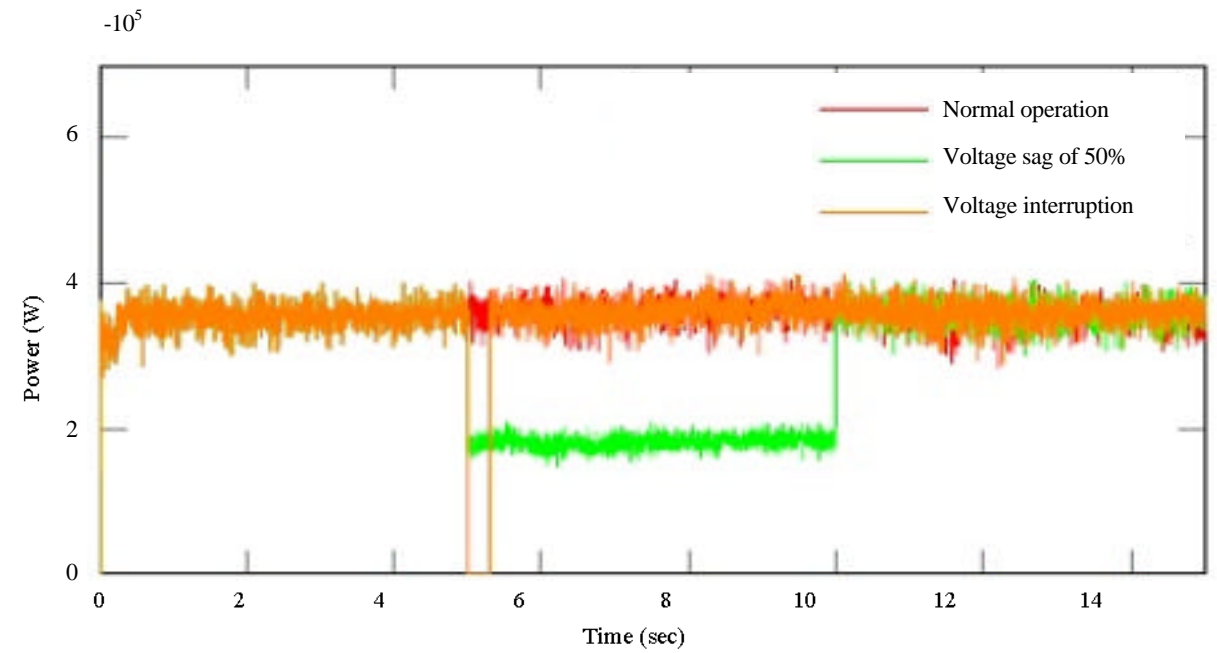

Fig. 15: Activation power

power grade then the produced energy maximized. Figure 12 present DC-bus voltages rest at its basic value with a small swing. Figure 13-16 show the grid line currents, active and reactive power, respectively. 


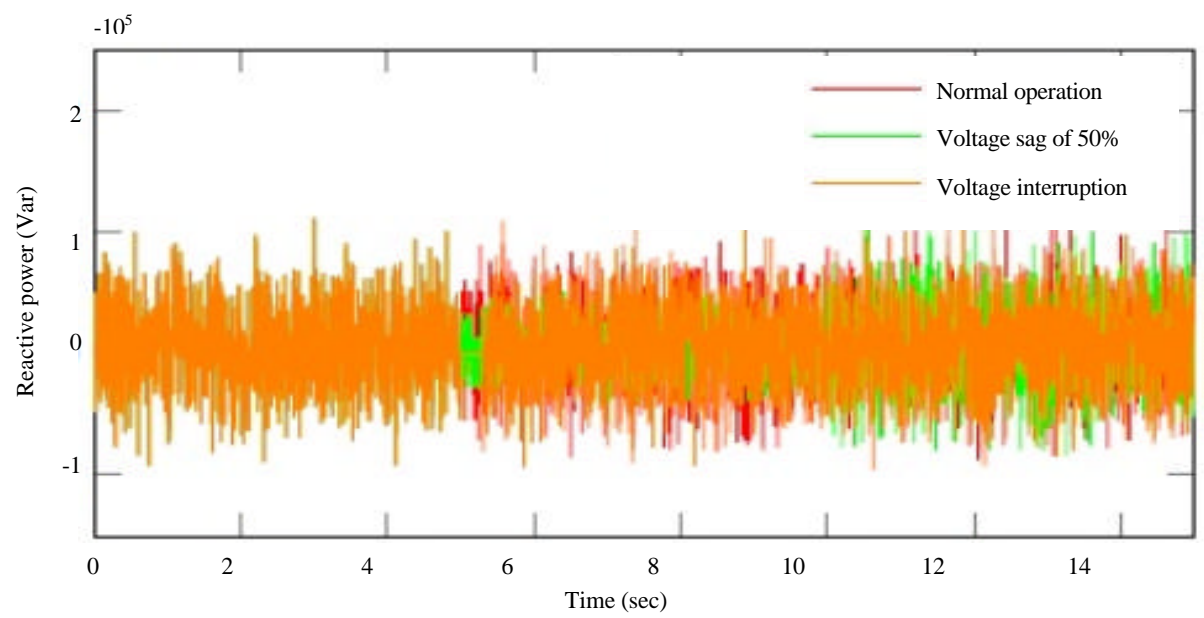

Fig. 16: Interactive power

\section{CONCLUSION}

Tidal energy is the result of the orbital energy of the Moon, Earth and Sun. Pollution ratios are close to zero of gases or radiations and are an inexhaustible source of energy, so, it is a kind of green energy. Tidal energy has some importance and advantages, since, it does not depend on season or weather, so, it is more predictable compared to other renewable energy sources such as wind energy or solar energy.

Explain characteristics that are important indicators in the design of water turbines for use in tidal energy and production characteristics and the number of blades in the water turbine. This study has proposed a strategy for controlling the dedicated network aspect to a permanent five-phase magnetic synchronous generator. Tidal turbines low voltage booster capacity. Power based on super capacitor, so, the storage system has been used for these purposes.

\section{SUGGESTIONS}

The proposed strategy is based on a predictive control model with the specified control set. The results achieved exhibit that the suggests control strategy is efficient in a condition of the preamble injection power in grid, the turbine system's ability to tide through the sags sharp grid effort.

\section{ACKNOWLEDGEMENTS}

I would like to thank my colleagues who helped me a lot in finalizing this project and for comments that provided insight and expertise that greatly improved the manuscript.

\section{REFERENCES}

Abd ALi, L.M. and H.A. Issa, 2018. Hybrid power generation using solar and wind energy. Young Sci., 7: 19-26.

Ansari, O.M., K.A. Mohmed and M.A.L. Ali, 2018. Design and Simulation a hybrid generation system through wind turbine and solar energy with a heat engine. Young Sci., 38: 11-24.

Astariz, S., A. Vazquez and G. Iglesias, 2015. Evaluation and comparison of the levelized cost of tidal, wave and offshore wind energy. J. Renewable Sustain. Energy, 7: 526-530.

Chen, H., N. At-Ahmed, M. Machmoum and M.E.H. Zam, 2016. Modeling and vector control of marine current energy conversion system based on doubly salient permanent magnet generator. IEEE. Trans. Sustainable Energy, 7: 409-418.

Efroimsky, M., 2018. Dissipation in a tidally perturbed body librating in longitude. Icarus, 306: 328-354.

Hashemi, M.R., S.P. Neill, P.E. Robins, A.G. Davies and M.J. Lewis, 2015. Effect of waves on the tidal energy resource at a planned tidal stream array. Renewable Energy, 75: 626-639.

Jahromi, M.J., A.I. Maswood and K.J. Tseng, 2013. Design and evaluation of a new converter control strategy for near-shore tidal turbines. IEEE. Trans. Ind. Electr., 60: 5648-5659. 
Signorelli, C., C. Villegas and J. Ringwood, 2012. Hardware-in-the-loop simulation of a heaving wave energy converter. Proceedings of the 9th European Conference on Wave and Tidal Energy (EWTEC), March 30, 2012, Maynooth University, Maynooth, Republic of Ireland, pp: $1-10$.
Tallam, R.M., G.L. Skibinski, T.A. Shudarek and R.A. Lukaszewski, 2011. Integrated differential-mode and common-mode filter to mitigate the effects of long motor leads on AC drives. IEEE. Trans. Ind. Appl., 47: 2075-2083.

Udo-Akuaibit, S.P., 2014. Morpho-dynamics shoreline offset at the entrance of Qua Iboe River Estuary, South East coast of Nigeria. J. Coast Dev., 17: 1-8. 